\title{
Oral bioavailability of quercetin from different quercetin glycosides in dogs
}

\author{
Marianne Reinboth ${ }^{1}$, Siegfried Wolffram ${ }^{2}$, Getu Abraham ${ }^{3}$, Fritz R. Ungemach ${ }^{3} \dagger$ and Rainer Cermak $^{1} *$ \\ ${ }^{1}$ Institute of Veterinary Physiology, University of Leipzig, An den Tierkliniken 7, 04103 Leipzig, Germany \\ ${ }^{2}$ Institute of Animal Nutrition and Physiology, University of Kiel, Kiel, Germany \\ ${ }^{3}$ Institute of Pharmacology, Pharmacy and Toxicology, University of Leipzig, Leipzig, Germany
}

(Received 14 October 2009 - Revised 6 January 2010 - Accepted 28 January 2010 - First published online 16 March 2010)

\begin{abstract}
Although the flavonol quercetin is used as a supplement in commercial dog food, data on quercetin bioavailability in dogs are not available. Thus, we investigated quercetin bioavailability (measured as area under the concentration-time curve) in nine adult beagle dogs at an oral dose of $10 \mathrm{mg} / \mathrm{kg}$ body weight (b.w.). The major fraction $(>80 \%)$ of flavonols circulating in blood plasma were conjugated metabolites of quercetin. The absolute bioavailability of quercetin (i.e. the fraction that reaches the systemic circulation) was only about $4 \%$. We also compared the oral bioavailability between the aglycone quercetin and its more often used glucorhamnoside (rutin) and 3-O-glucoside (isoquercitrin) at an equimolar dose of $30 \mu \mathrm{mol} / \mathrm{kg}$ b.w. (corresponding to $10 \mathrm{mg}$ quercetin $/ \mathrm{kg}$ ). Quercetin and isoquercitrin were mainly absorbed in the small intestine with isoquercitrin being one and a half times more bioavailable than quercetin. Maximal plasma concentration after isoquercitrin treatment was $0 \cdot 89$ (SEM 0.07) $\mu \mathrm{mol} / \mathrm{l}$. Although quercetin absorption from rutin was delayed, relative bioavailability was not lower than from the aglycone itself. The latter observation is in clear contrast to findings in human subjects, pigs or rats and might indicate that rutin is a better source of quercetin in dogs than in other species. However, potential in vivo quercetin effects beyond the gastrointestinal tract are limited by the intensive metabolism as well as by the rather low bioavailability of this flavonol.
\end{abstract}

Quercetin glycosides: Quercetin: Dogs: Bioavailability: Flavonoids

Plant flavonoids including the flavonol quercetin have become increasingly popular as dietary supplements in recent years. These compounds have been suggested to have various beneficial effects like preventing cancer, suppressing inflammation or decreasing fat absorption ${ }^{(1)}$. Quercetin, an effective antioxidant in vitro, is added to certain commercial dog foods and marketed as a compound preventing or improving a multitude of age-related diseases that are associated with increased oxidative stress. In order to evaluate potential effects of such compounds in vivo, a sound knowledge of their bioavailability and metabolism is prerequisite. In rats and pigs, the absolute bioavailability (i.e. the fraction of an ingested compound that reaches the systemic circulation) of unchanged quercetin was only $5 \%$ and $<1 \%$, respectively ${ }^{(2,3)}$. However, the major fraction of plasma metabolites with an intact flavonol structure consists of methylated, glucuronidated and sulphated derivatives of quercetin $^{(4-6)}$. Taking also these metabolites into consideration, the absolute bioavailability of quercetin was noticeably higher with 59 and $17 \%$ for rats and pigs, respectively ${ }^{(2,3)}$. Peak plasma concentrations after oral quercetin doses of 10 or $50 \mathrm{mg} / \mathrm{kg}$ body weight (b.w.) were in the low $\mu \mathrm{mol} / 1$ range in these studies. This was also true for human subjects that ingested up to $200 \mathrm{mg}$ of the flavonol in a single dose ${ }^{(7)}$.
In plants, flavonols like quercetin occur mainly as $O$-glycosides and are linked to sugars like glucose, galactose or rhamnose ${ }^{(8)}$. Supplemented commercial dog food also contains such glycosides like quercetin-3-O-glucorhamnoside (rutin). The glycosidic structure has a large impact on quercetin bioavailability, because the hydrolytic release of the sugarfree aglycone quercetin is a prerequisite for its absorption. Certain glycosides like quercetin-3-O-glucoside (isoquercitrin) are substrates for the small intestinal brush border enzyme lactase-phlorizin hydrolase (EC 3.2.1.108) ${ }^{(9,10)}$. Quercetin can be enzymatically released from isoquercitrin and is, thus, absorbed mainly in the small intestine. This results in a relatively high bioavailability of quercetin from isoquercitrin. In contrast, the 3-O-glucorhamnoside of quercetin is not a substrate of lactase-phlorizin hydrolase. Release of quercetin aglycone depends on hydrolysis by bacterial $\alpha$-rhamnosidases and $\beta$-glucosidases in the lower gastrointestinal tract. Thus, absorption of quercetin from rutin is delayed, and quercetin bioavailability is much smaller than from the aglycone itself, at least in human subjects, rats and pigs ${ }^{(11-14)}$.

The dog is a commonly used animal model for pharmacokinetic and metabolic studies ${ }^{(15)}$. Previously, dogs have been used to study flavonoid toxicity ${ }^{(16)}$. Similar to human subjects, aged dogs are prone to diseases like cancer, diabetes

\footnotetext{
Abbreviations: AUC, area under the plasma concentration-time curve; b.w., body weight; i.v., intravenous; MRT, mean residence time; $T_{\text {max }}$, times to achieve maximum plasma concentrations.

* Corresponding author: Rainer Cermak, fax +4934197 38028, email cermak@vetmed.uni-leipzig.de

$\dagger$ Deceased.
} 
mellitus or cardiac insufficiency. Manufacturers of pet foods increasingly supplement diets with quercetin and quercetin glycosides and claim preventive or therapeutic effects against those diseases by their supplementation. In spite of its current use in dog food, no information is available about quercetin bioavailability from the aglycone or from glycosides like rutin for this particular species. Thus, we investigated the bioavailability of quercetin in Beagle dogs. We administered oral doses of $30 \mu \mathrm{mol} / \mathrm{kg}$ b.w. of the aglycone, isoquercitrin or rutin, equivalent to $10 \mathrm{mg}$ quercetin $/ \mathrm{kg}$ b.w. Daily intakes of $5-10 \mathrm{mg}$ quercetin $/ \mathrm{kg} \mathrm{b.w}$. can be realised by feeding currently available dog foods that are supplemented with quercetin or rutin.

\section{Experimental methods}

\section{Chemicals}

Quercetin, isoquercitrin, rutin, tamarixetin, isorhamnetin and rhamnetin were obtained from Roth, Karlsruhe, Germany; all compounds were of HPLC grade. $\beta$-Glucuronidase/ sulphatase (crude enzyme extract from Helix pomatia) and other chemicals were from Sigma-Aldrich AG, Deisenhofen, Germany.

\section{Animals and experiments}

Nine adult 6-year-old Beagle dogs of mixed sex (three castrated males, six females) with a mean b.w. of $14 \cdot 2$ (SEM $0.7) \mathrm{kg}$ were used. All animal experiments were in compliance with national laws. They were approved by the Animal Welfare Officer of the Faculty of Veterinary Medicine, University of Leipzig, and by the relevant legal authorities (Regierungspräsidium Leipzig: TVV-Nr. 15/06). All flavonols were orally administered at a dose of $30 \mu \mathrm{mol} / \mathrm{kg} \mathrm{b.w.} \mathrm{after}$ a fasting period of $24 \mathrm{~h}$. Flavonols were mixed into $20 \mathrm{~g}$ of a commercial flavonol-free dog food based on chicken meat ('Premium Spurty', Penny Markt, Köln, Germany). This test meal contained (per $\mathrm{kg}$ diet) $99 \mathrm{~g}$ crude protein, $55 \mathrm{~g}$ crude fat, $5 \mathrm{~g}$ crude fibre, $16 \mathrm{~g}$ crude ash, $188 \mathrm{~g}$ DM. Complete voluntary ingestion of the test meal was controlled under supervision. Blood samples from forearm and hind leg veins were collected directly before flavonol intake and after ingestion of the test meals over a period of up to $72 \mathrm{~h}$. Each dog received the three flavonols quercetin, isoquercitrin and rutin in a Latin square design (three dogs per group). Between each single test meal was a wash-out period of at least 2 weeks. Due to drop outs during blood sampling, only seven dogs were included in the evaluation. In order to determine the absolute bioavailability of quercetin, eight dogs received the flavonol orally $(30 \mu \mathrm{mol} / \mathrm{kg}$ b.w.) as described before and intravenously $(1.5 \mu \mathrm{mol} / \mathrm{kg}$ b.w., solved in dimethyl sulphoxide) in a cross-over design. There was a 2-week wash-out period between these treatments.

\section{Processing of blood samples and HPLC analysis}

Blood samples $(3 \mathrm{ml})$ were drawn into heparinised containers and immediately centrifuged $\left(1500 \mathrm{~g}, 10 \mathrm{~min}, 4^{\circ} \mathrm{C}\right)$. Plasma was stored at $-70^{\circ} \mathrm{C}$ until analysis. Further processing was performed as described previously ${ }^{(13)}$. Aliquots of each plasma sample were spiked with $30 \mu \mathrm{g}$ rhamnetin in $10 \mu \mathrm{l}$ methanol (internal standard). They were treated with $5 \mu \mathrm{Kat} / \mathrm{l}$ $\beta$-glucuronidase $/ 72 \mu \mathrm{Kat} / 1$ sulphatase (crude enzyme extract from $H$. pomatia) for cleavage of all ester bonds of glucuronides/sulphates. In order to analyse only the unconjugated flavonols, aliquots of the same samples were incubated with water instead of the enzyme extract. Flavonols were analysed by HPLC as described previously ${ }^{(2)}$. Calibration curves for quercetin, kaempferol, isorhamnetin and tamarixetin were obtained by the addition of these flavonols from methanolcontaining stock solutions to flavonol-free dog plasma, with rhamnetin serving as an internal standard. All the samples were corrected for contamination with quercetin and kaempferol by the crude enzyme extract.

\section{Pharmacokinetic calculations}

Maximum plasma concentrations and times to achieve maximum plasma concentrations $\left(T_{\max }\right)$ were obtained from the observed data. The terminal elimination rate constant $\left(\lambda_{z}\right)$ was calculated by linear regression of the terminal slope of the logarithm of plasma concentration $v$. time profile. For calculations of area under the plasma concentration-time curves (AUC), the linear trapezoidal rule was used with extrapolation to infinity $\left(\mathrm{AUC}_{0 \rightarrow \infty}\right)$. The mean residence time (MRT) was calculated from the ratio of the area under the first moment curve and the AUC. Pharmacokinetic calculations were performed with the software WinNonlin 5.1 (Pharsight Corporation, St Louis, MO, USA). Absolute bioavailability $(F)$ was calculated by the dose-corrected ratio of the AUC values after oral and intravenous (i.v.) administration with the following formula:

$$
F=\frac{\text { AUC }_{\text {oral }}}{\text { AUC }_{\text {i.v. }}} \times \frac{\text { Dose }_{\text {i.v. }}}{\text { Dose }_{\text {oral }}} \times 100 \% .
$$

\section{Statistics}

Data are presented as mean values with their standard errors (SD in figures). Values from two groups were compared with the Student's $t$ test, values obtained with the different flavonols were compared using a one-way ANOVA model (repeated measures) followed by the Bonferroni post-test integrated in GraphPad Prism ${ }^{\mathrm{TM}}$ version 5 (GraphPad Software, San Diego, CA, USA). Differences were considered significant when $P<0 \cdot 05$.

\section{Results}

After oral intake of quercetin $(10 \mathrm{mg} / \mathrm{kg}$ b.w.), the flavonol was rapidly absorbed, and it appeared within $15 \mathrm{~min}$ after ingestion in blood plasma (Fig. 1). The major fraction of plasma flavonols consisted of glucuronidated and/or sulphated conjugates that were only detectable after enzymatic hydrolysis of the blood samples before analysis. Free quercetin also appeared to a certain extent in the circulatory system. Based on the comparison of AUC values with and without enzymatic pretreatment of the samples, free quercetin aglycone represented about $16 \%$ of total plasma quercetin including conjugates. Besides quercetin, its methylated metabolite isorhamnetin $\left(3^{\prime}-O\right.$-methyl-quercetin) was also detected. 


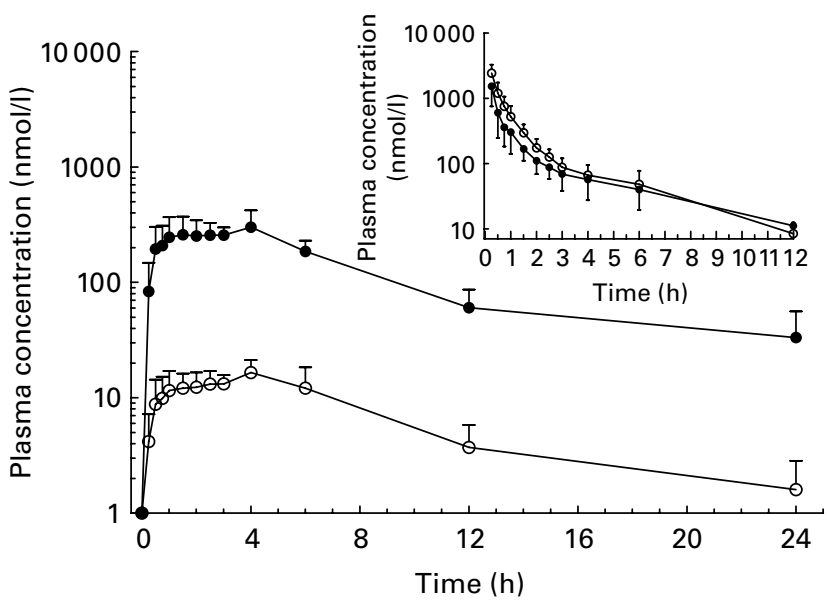

Fig. 1. Time $v$. plasma concentration curves of quercetin $(\bullet)$ and isorhamnetin $(\bigcirc)$ after oral administration of quercetin $(10 \mathrm{mg} / \mathrm{kg}$ body weight (b.w.)) to eight beagle dogs. Inset: time $v$. plasma concentration curves of the same flavonols after intravenous (i.v.) administration of quercetin $(0.5 \mathrm{mg} / \mathrm{kg} \mathrm{b.w.)}$. Concentrations at time 0 after i.v. administration were extrapolated. Blood samples were enzymatically pretreated; data are expressed as mean values and standard deviations.

Plasma concentrations of isorhamnetin were one order of magnitude lower than those of quercetin (Fig. 1). Based on AUC values, isorhamnetin represented about $10 \%$ of all the metabolites with an intact flavonol structure. Tamarixetin (4'-O-methyl-quercetin), another methylated quercetin metabolite that is found in several species after ingestion of quercetin, was rarely detected in amounts usually below its limit of quantification (i.e. $2.5 \mathrm{nmol} / \mathrm{l}$ ). Kaempferol, a flavonol with one hydroxyl group less than quercetin in its C-ring, represented only about $2 \%$ of all detected metabolites in dog plasma (data not shown).

In contrast, plasma concentrations of isorhamnetin exceeded quercetin concentrations after i.v. administration of quercetin; this was obvious already after $15 \mathrm{~min}$ (Fig. 1, inset). Concentrations of the methylated metabolite continued to be higher than the respective quercetin concentrations up to $6 \mathrm{~h}$ Thus, AUC values for isorhamnetin were about $40 \%$ higher than those for quercetin after i.v. administration (Table 1). Taking all plasma metabolites with an intact flavonol structure into consideration, absolute bioavailability of quercetin was about $4 \%$ (Table 1 ).

After ingestion of an equimolar dose of its 3-O-glucoside isoquercitrin, plasma concentrations of quercetin were significantly higher than those after ingestion of the aglycone (Fig. 2). Two plasma peaks were apparent, a first one within the first hour and a second peak $4 \mathrm{~h}$ after ingestion of the flavonol that was higher than the first one. A similar situation was observed after administration of quercetin aglycone, although the two peaks were not quite as distinctly visible. Quercetin plasma concentration stayed on the same level after the first peak until it reached a maximum concentration after $4 \mathrm{~h}$ (second peak). While the second peak appeared at the same time after both treatments, $T_{\max }$ of the first peak was significantly shorter after administration of isoquercitrin (Table 2). Forty-eight hours after administration of both quercetin and isoquercitrin, plasma flavonol concentrations were at the limit of quantification (i.e. $2 \cdot 5 \mathrm{nmol} / \mathrm{l}$ ).
Table 1. Absolute bioavailability of quercetin and area under the plasma concentration-time curve $\left(\mathrm{AUC}_{0 \rightarrow \infty}\right)$ values of the main flavonol metabolites after oral and intravenous (i.v.) administration of quercetin to dogs $(n 8)$

(Mean values with their standard errors)

\begin{tabular}{|c|c|c|}
\hline & Mean & SEM \\
\hline i.v. Dose $(\mathrm{mg} / \mathrm{kg})$ & 0.5 & \\
\hline $\mathrm{AUC}_{\text {Quercetin }}(\mathrm{min} \times \mu \mathrm{mol} / \mathrm{I})$ & $113 \cdot 2$ & $12 \cdot 7$ \\
\hline $\mathrm{AUC}_{\text {Isorhamnetin }}(\mathrm{min} \times \mu \mathrm{mol} / \mathrm{l})$ & $160 \cdot 2$ & $17 \cdot 2$ \\
\hline $\mathrm{AUC}_{\mathrm{Kaempferol}}(\min \times \mu \mathrm{mol} / \mathrm{l})$ & $6 \cdot 1$ & 0.6 \\
\hline $\mathrm{AUC}_{\text {Total }}(\min \times \mu \mathrm{mol} / \mathrm{l})$ & $279 \cdot 4$ & $27 \cdot 1$ \\
\hline Oral dose $(\mathrm{mg} / \mathrm{kg})$ & 10 & \\
\hline $\mathrm{AUC}_{\text {Quercetin }}(\min \times \mu \mathrm{mol} / \mathrm{l})$ & $185 \cdot 0$ & $10 \cdot 1$ \\
\hline $\mathrm{AUC}_{\text {Isorhamnetin }}(\min \times \mu \mathrm{mol} / \mathrm{l})$ & $9 \cdot 8$ & $1 \cdot 3$ \\
\hline $\mathrm{AUC}_{\mathrm{Kaempferol}}(\min \times \mu \mathrm{mol} / \mathrm{l})$ & $5 \cdot 8$ & $1 \cdot 1$ \\
\hline $\mathrm{AUC}_{\text {Total }}(\min \times \mu \mathrm{mol} / \mathrm{l})$ & $200 \cdot 6$ & $11 \cdot 8$ \\
\hline$F(\%)$ & $3 \cdot 8$ & 0.4 \\
\hline
\end{tabular}

$A U C_{\text {Quercetin }}, A U C$ values for quercetin; $A U C_{\text {Isorhamnetin }}, A U C$ values for isorhamnetin; $A \cup C_{\text {Kaempferol, }} A U C$ values for kaempferol; $A \cup C_{\text {Total }}$, sum of $A U C$ values for all three flavonols; $F$, absolute bioavailability.

Absorption of quercetin from rutin was considerably delayed. Within the first hour after ingestion, plasma concentrations hardly exceeded $10 \mathrm{nmol} / \mathrm{l}$. Peak concentrations were reached $11 \mathrm{~h}$ after ingestion of the glycoside, which was significantly later than after the two other treatments (Fig. 2 and Table 2). The following decrease in plasma concentration was also slower compared with the administration of either isoquercitrin or quercetin. This correlated with a significantly longer MRT for quercetin after ingestion of rutin. While AUC values of quercetin, isorhamnetin and of total plasma flavonols were significantly higher after administration of isoquercitrin, the respective values after quercetin and rutin treatment did not differ (Table 2).

\section{Discussion}

In order to estimate the oral bioavailability of quercetin in dogs, we determined the area under the plasma concentration-time curves of its main metabolites.

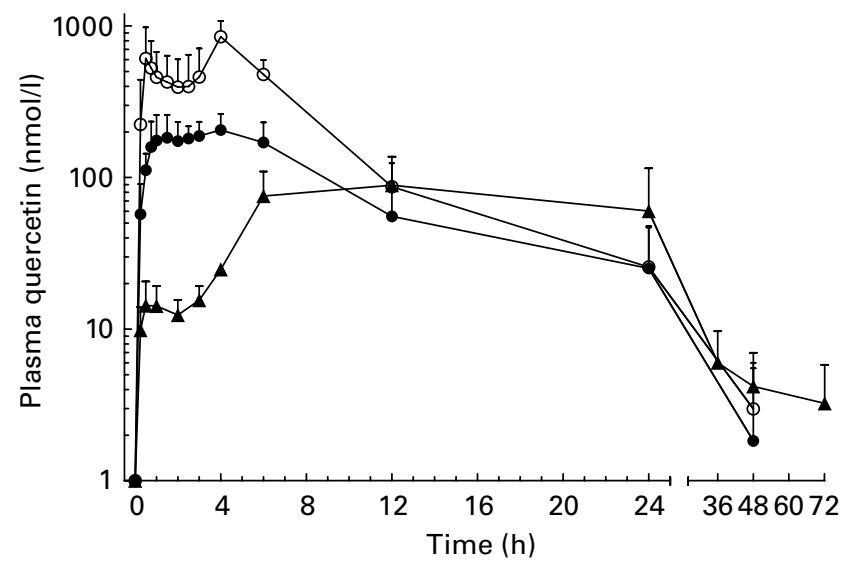

Fig. 2. Time $v$. plasma concentration curves of quercetin after oral administration of equimolar amounts ( $30 \mu \mathrm{mol} / \mathrm{kg}$ body weight) of quercetin $(\bullet)$, isoquercitrin $(O)$ or rutin $(\boldsymbol{\Lambda})$ to seven beagle dogs. Blood samples were enzymatically pretreated; data are expressed as mean values and standard deviations. 
Table 2. Pharmacokinetic parameters of the plasma metabolite quercetin and area under the plasma concentration-time curve $\left(\mathrm{AUC}_{0 \rightarrow \infty}\right)$ values for the main metabolites after oral administration of quercetin, isoquercitrin or rutin (each $30 \mu \mathrm{mol} / \mathrm{kg}$ ) to dogs

(Mean values with their standard errors)

\begin{tabular}{|c|c|c|c|c|c|c|}
\hline \multirow[b]{2}{*}{ Pharmacokinetic parameter } & \multicolumn{2}{|c|}{ Quercetin } & \multicolumn{2}{|c|}{ Isoquercitrin } & \multicolumn{2}{|c|}{ Rutin } \\
\hline & Mean & SEM & Mean & SEM & Mean & SEM \\
\hline$C_{\max 1}(\mathrm{nmol} / \mathrm{l})$ & 233.6 & $31 \cdot 3$ & $667 \cdot 4^{\star \star \star}$ & $131 \cdot 2$ & $17 \cdot 0$ & $2 \cdot 2$ \\
\hline$T_{\max 1}(\mathrm{~h})$ & $1 \cdot 2^{\star *}$ & 0.1 & 0.8 & 0.1 & 0.7 & 0.1 \\
\hline$C_{\max 2}(\mathrm{nmol} / \mathrm{l})$ & $229 \cdot 2$ & $18 \cdot 2$ & $888 \cdot 3^{\star \star \star}$ & $71 \cdot 0$ & $113 \cdot 7$ & $18 \cdot 1$ \\
\hline$T_{\max 2}(\mathrm{~h})$ & 3.9 & 0.5 & $4 \cdot 1$ & 0.3 & $11 \cdot 1^{\star}$ & $2 \cdot 4$ \\
\hline MRT (h) & $7 \cdot 8$ & 0.6 & $6 \cdot 4$ & 0.5 & $18 \cdot 0^{\star \star \star}$ & 1.0 \\
\hline $\mathrm{AUC}_{\text {Quercetin }}(\min \times \mu \mathrm{mol} / \mathrm{I})$ & $155 \cdot 2$ & $17 \cdot 4$ & $353 \cdot 4^{* * *}$ & $21 \cdot 3$ & $131 \cdot 2$ & $20 \cdot 0$ \\
\hline $\mathrm{AUC}_{\text {Isorhamnetin }}(\mathrm{min} \times \mu \mathrm{mol} / \mathrm{l})$ & 11.4 & 1.9 & $44 \cdot 9^{* \star}$ & 7.9 & $17 \cdot 0$ & 1.9 \\
\hline $\mathrm{AUC}_{\text {Kaempferol }}(\mathrm{min} \times \mu \mathrm{mol} / \mathrm{l})$ & 8.2 & 1.5 & 11.9 & $1 \cdot 0$ & 11.8 & 0.9 \\
\hline $\mathrm{AUC}$ Total $(\min \times \mu \mathrm{mol} / \mathrm{l})$ & 174.9 & $19 \cdot 7$ & $410 \cdot 2^{\star \star *}$ & $26 \cdot 7$ & $160 \cdot 0$ & $21 \cdot 3$ \\
\hline Relative bioavailability (\%) & 100 & & 234.5 & & 91.5 & \\
\hline
\end{tabular}

$C_{\max }$, maximal plasma concentration, subscripts 1 and 2 denote first and second plasma peak, respectively; $T_{\max }$, time to reach $C_{\text {max }}$; MRT, mean residence time; $A_{U} C_{\text {Quercetin, }}$ AUC values for quercetin; $A U C_{\text {lsorhamnetin }}$, AUC values for isorhamnetin; $A \cup C_{\text {Kaempferol }}$, $A U C$ values for kaempferol; $A U C_{\text {Total }}$, sum of $A U C$ values for all three flavonols.

Mean values were significantly different from values in other columns with ${ }^{\star} P<0.05$, ${ }^{\star \star} P<0.01$, or ${ }^{\star * \star} P<0.001$, respectively $(n 7)$

Plasma pharmacokinetic data after oral administration of flavonoids do not provide direct information about the absorbed amount, though. Analysing the cumulative amount of urinary flavonoid excretion provides an estimate of the minimal absorbed amount. One disadvantage of this method is that other excretory routes are not considered. It has been demonstrated that up to $40 \%$ of the absorbed amount of quercetin is eliminated via bile during first-pass metabolism ${ }^{(17)}$. We administered quercetin intravenously to get a reference AUC value that is, by definition, after i.v. administration $100 \%$. This is the standard method for determining the bioavailability of xenobiotics. It has not been performed for determining quercetin bioavailability in human subjects for obvious reasons. This approach considers blood as the relevant compartment for potential flavonoid effects beyond the alimentary tract. In our opinion, this is the best method due to the lack of reliable markers of this flavonol's metabolic utilisation in vivo ${ }^{(18)}$.

In our experiments, using a quercetin dose that is reached by feeding commercial quercetin-supplemented dog food, absolute bioavailability of the flavonol was only about $4 \%$, even when all the conjugated metabolites with an intact flavonol structure were included. A single oral dose of $150 \mathrm{mg}$ quercetin in a beagle weighing $15 \mathrm{~kg}$ corresponded to plasma peak levels below $1 \mu \mathrm{mol} / \mathrm{l}$.

Similar to other species, the major part of circulating quercetin (i.e. more than $80 \%$ based on AUC values) consisted of glucuronidated and/or sulphated metabolites. Isorhamnetin represented about $10 \%$ of all the metabolites after oral administration. Interestingly, this methylated metabolite constituted more than $50 \%$ of plasma flavonols after i.v. administration. This surprising observation could be explained by a high hepatic methylation rate of quercetin provided that it reaches the liver via arterial blood in its native form as is the case when administered intravenously. In contrast, intensive first-pass glucuronidation and sulphation of ingested quercetin in the intestinal mucosa will probably prevent a subsequent methylation of the flavonol.
To date, only a few studies have investigated absorption and bioavailability of flavonoids in dogs. Recently, a mixture of grapefruit flavanones (naringin, narirutin and naringenin) was orally administered to adult beagle dogs with a mean b.w. of $14 \mathrm{~kg}^{(19)}$. The dose of $70 \mathrm{mg}$ that each dog received in that study was approximately half of that we used in our trial $(10 \mathrm{mg} / \mathrm{kg}$ b.w.). Interestingly, the measured plasma concentrations in that study were also in the nanomolar range. The median maximum plasma concentrations of the most abundant metabolite, naringenin, was only $238 \mathrm{nmol} / \mathrm{l}$. The respective $T_{\max }$ and MRT values were $80 \mathrm{~min}$ and $3.3 \mathrm{~h}$, respectively, for this flavanol. Besides the shorter MRT for naringenin, these parameters are comparable to the ones for the flavonol quercetin in our trial. The oral bioavailability of 5-methoxyflavone was investigated in another study using rats and $\log \mathrm{s}^{(20)}$. Because the focus in the latter study was more on the development of the analytical method, only two beagle dogs were used that received a flavone dose of $10 \mathrm{mg} / \mathrm{kg}$. The oral bioavailability of 5-methoxyflavone was 72 and $33 \%$ in these two $\operatorname{dogs}{ }^{(20)}$.

In many food supplements, naturally occurring glycosides of flavonoids are used. It is known that the type of glycoside has a large impact on bioavailability. The 3-O-glucoside (isoquercitrin) and $4^{\prime}$ - $\mathrm{O}$-glucoside of quercetin are already absorbed in the small intestine and demonstrate a relatively high bioavailability ${ }^{(12,13)}$. Bioavailability of isoquercitrin was $50-180 \%$ higher than that of quercetin in pigs and rats $^{(13,14)}$. In comparison, bioavailability of isoquercitrin was $134 \%$ higher than that of the aglycone in our beagle dogs. Although absorption from isoquercitrin was reasonably fast with a $T_{\max }$ value of $<1 \mathrm{~h}$, plasma levels of quercetin after administration of the 3-O-glucoside had a distinct second peak about $4 \mathrm{~h}$ after ingestion which seems to indicate an intensive enterohepatic circulation of the metabolites. This was very similar to our previous observation in pigs ${ }^{(13)}$.

In contrast to quercetin glucosides, the abundant rutin is mainly absorbed in lower parts of the intestinal tract, because bacterial $\alpha$-rhamnosidases and $\beta$-glucosidases are necessary to 
release the aglycone from this 3 - $O$-glucorhamnoside. This corresponds to $T_{\max }$ values as long as $6 \mathrm{~h}$ in human subjects or $3.5 \mathrm{~h}$ in pigs ${ }^{(12,13)}$. Similarly, quercetin absorption from rutin was also delayed in dogs with a mean $T_{\max }$ of $11 \mathrm{~h}$. However, in the lower intestinal tract, a significant part of the released quercetin is not absorbed because it is further degraded by bacterial enzymes ${ }^{(21,22)}$. Thus, bioavailability of quercetin from rutin is generally low. In rats and pigs, relative bioavailability from rutin was only $25 \%$ compared with the aglycone $^{(13,14)}$.

Surprisingly, in dogs, the relative bioavailability from rutin was not substantially lower than that from quercetin. This can be explained by the observation that plasma concentrations of the flavonol decreased at a slower rate after administration of the glucorhamnoside. MRT was significantly longer after rutin administration compared with the other two treatments. We speculate that the bacterial degradation of the released quercetin in the hindgut of dogs could be less efficient than in the previously investigated species. Using caecal content from pigs in a colon simulation technique, we previously observed that the rate of bacterial quercetin release from rutin was smaller than the degradation rate of the released quercetin ${ }^{(22)}$. In comparison with human subjects, dogs have a much shorter transit time in the large intestine, and fermentation efficiency is very low in comparison with human subjects, pigs or rats $^{(23,24)}$. We hypothesise that the large intestinal microflora of this carnivore species is less adapted to metabolise plant flavonoids. If bacterial degradation of quercetin in the hindgut was less efficient in dogs than in pigs, a larger part of the released flavonol would be available for absorption in the former species. This could explain the relatively high rutin bioavailability in dogs and should be further investigated. Nevertheless, in dogs, rutin seems to be a much better quercetin source than in other species. Because most dogs are usually fed once a day, the rather long MRT of quercetin after rutin could be important for the maintenance of relevant flavonol concentrations in plasma (and tissues) when administered with the regular meals.

With our analytical method, we could not identify specific molecular structures of the conjugated metabolites. Other groups identified a number of quercetin and isorhamnetin conjugates in plasma, body tissues and urine using liquid chromatography-MS ${ }^{(4-6)}$. Quercetin-3-glucuronide and isorhamnetin-3-glucuronide were detected as the main metabolites in plasma of human subjects who had ingested tomato juice containing rutin ${ }^{(5)}$. In addition to these two glucuronides, further nine quercetin metabolites were, at least partially, identified in the plasma of human subjects after the ingestion of onions rich in quercetin glycosides ${ }^{(6)}$. Among those nine metabolites, quercetin- $3^{\prime}$-sulphate, a quercetin diglucuronide and a quercetin glucuronide sulphate were the most prevalent ones. Additional metabolites were detected in urine ${ }^{(5,6)}$. In the plasma of rats fed a quercetin-containing diet, several glucuronidated, sulphated and methylated quercetin metabolites were detected, although the exact position of the functional groups was not identified. A methylated quercetin monoglucuronide sulphate was the most abundant plasma metabolite in these rats ${ }^{(4)}$. To our knowledge, specific structures of quercetin and isorhamnetin conjugates in dog plasma that comprise the majority of quercetin metabolites in this species have not been identified yet. Due to the known species differences with regard to xenobiotic-metabolising enzyme activities, we expect that the metabolite pattern in dogs might be different from that shown previously in human subjects ${ }^{(25,26)}$.

Some authors question the accuracy of flavonoid analyses that use a glucuronidase/sulphatase preparation step as performed by us ${ }^{(27)}$. Indeed, a study that compared isoflavone contents in several rat body tissues using either enzymatic or acid hydrolysis of isoflavone conjugates before liquid chromatography-MS found significantly higher isoflavone concentrations after acid hydrolysis ${ }^{(28)}$. The authors hypothesised that encapsulation of the conjugates in microsomes, binding of isoflavones to specific tissue proteins (like oestrogen receptors) and/or the occurrence of tissue components that inhibit $\beta$-glucuronidase or sulphatase activity could explain the significantly lower isoflavone recovery after enzymatic hydrolysis. Interestingly, they found no differences in isoflavone concentrations in blood serum after enzymatic or acid hydrolysis ${ }^{(28)}$. We assume that factors responsible for limiting enzymatic isoflavone conjugate hydrolysis in body tissues may have no significance in blood. Thus, we think that the accuracy of the analysed plasma concentrations in our study is acceptable.

A daily quercetin dose of $10 \mathrm{mg} / \mathrm{kg}$ can be achieved by feeding commercially available quercetin-supplemented dog food. From rat studies, it can be concluded that chronically ingested quercetin is generally non-toxic, even when administered at much higher dietary concentrations ${ }^{(29)}$. Interestingly, a similar dose of $150 \mathrm{mg}$ per animal of another flavonoid, the abundant tea flavanol epigallocatechin gallate, was able to induce intestinal toxicity and hepatotoxicity and even caused fatalities in beagle dogs when administered for several weeks $^{(16)}$. This toxic potential of epigallocatechin gallate is probably due to the fact that the plasma fraction of native unconjugated epigallocatechin gallate is much higher because it is less metabolised than quercetin. However, one should be careful in extrapolating results of toxicity studies conducted with rodents to other species like dogs.

In summary, we have shown that absolute bioavailability of quercetin was only $4 \%$ in dogs. As in other species like human subjects, rats and pigs, the oral bioavailability of quercetin after the application of the quercetin monoglucoside isoquercitrin was significantly higher compared with the application of the aglycone. When the glycoside rutin was used as a quercetin source, flavonol absorption was delayed. In contrast to other investigated species, relative quercetin bioavailability from rutin was not smaller than from quercetin aglycone itself. However, the intensive metabolism of quercetin as well as the low absolute bioavailability of the flavonol has to be taken into account when potential in vivo effects of the flavonol are evaluated in this species.

\section{Acknowledgements}

We would like to thank Dr B. Fürll, K. Benson and P. Philipp for their help with blood sampling. Authors state that no conflicts of interest exist. The present study had no external funding. M. R. performed the experiments, sample analysis and data evaluation, and was involved in study design and manuscript composition; S. W., G. A. and F. R. U. were involved in study design and manuscript revision; R. C. was involved in study design, data evaluation and manuscript composition. 


\section{References}

1. Yang CS, Sang S, Lambert JD, et al. (2008) Bioavailability issues in studying the health effects of plant polyphenolic compounds. Mol Nutr Food Res 52, S139-S151.

2. Ader P, Wessmann S \& Wolffram S (2000) Bioavailability and metabolism of the flavonol quercetin in the pig. Free Radic Biol Med 28, 1056-1067.

3. Chen X, Yin OQ, Zuo Z, et al. (2005) Pharmacokinetics and modeling of quercetin and metabolites. Pharm Res 22, 892-901.

4. Graf BA, Ameho C, Dolnikowski GG, et al. (2006) Rat gastrointestinal tissues metabolize quercetin. J Nutr 136, 39-44.

5. Jaganath IB, Mullen W, Edwards CA, et al. (2006) The relative contribution of the small and large intestine to the absorption and metabolism of rutin in man. Free Radic Res 40, 1035-1046.

6. Mullen W, Edwards CA \& Crozier A (2006) Absorption, excretion and metabolite profiling of methyl-, glucuronyl-, glucosyl- and sulpho-conjugates of quercetin in human plasma and urine after ingestion of onions. Br J Nutr 96, 107-116.

7. Manach C, Williamson G, Morand C, et al. (2005) Bioavailability and bioefficacy of polyphenols in humans. I. Review of 97 bioavailability studies. Am J Clin Nutr 81, 230S-242S

8. Bravo L (1998) Polyphenols: chemistry, dietary sources, metabolism, and nutritional significance. Nutr Rev 56, 317-333.

9. Day AJ, Dupont MS, Ridley S, et al. (1998) Deglycosylation of flavonoid and isoflavonoid glycosides by human small intestine and liver beta-glucosidase activity. FEBS Lett 436, 71-75.

10. Ioku K, Pongpiriyadacha Y, Konishi Y, et al. (1998) Betaglucosidase activity in the rat small intestine toward quercetin monoglucosides. Biosci Biotechnol Biochem 62, 1428-1431.

11. Manach C, Morand C, Demigné C, et al. (1997) Bioavailability of rutin and quercetin in rats. FEBS Lett 409, 12-16.

12. Hollman PCH, Bijsman MNCP, van Gameren Y, et al. (1999) The sugar moiety is a major determinant of the absorption of dietary flavonoid glycosides in man. Free Radic Res 31, $569-573$.

13. Cermak R, Landgraf S \& Wolffram S (2003) The bioavailability of quercetin in pigs depends on the glycoside moiety and on dietary factors. J Nutr 133, 2802-2807.

14. Morand C, Manach C, Crespy V, et al. (2000) Quercetin 3-O-beta-glucoside is better absorbed than other quercetin forms and is not present in rat plasma. Free Radic Res 33, 667-676.

15. Tibbitts $\mathbf{J}$ (2003) Issues related to the use of canines in toxicologic pathology - issues with pharmacokinetics and metabolism. Toxicol Pathol 31, 17-24.
16. Isbrucker RA, Edwards JA, Wolz E, et al. (2006) Safety studies on epigallocatechin gallate (EGCG) preparations. Part 2: dermal, acute and short-term toxicity studies. Food Chem Toxicol 44, 636-650.

17. Crespy V, Morand C, Besson C, et al. (2003) The splanchnic metabolism of flavonoids highly differed according to the nature of the compound. Am J Physiol 284, G980-G988.

18. Cermak R, Durazzo A, Maiani G, et al. (2009) The influence of postharvest processing and storage of foodstuffs on the bioavailability of flavonoids and phenolic acids. Mol Nutr Food Res 53, S184-S193.

19. Mata-Bilbao M, Andrés-Lacueva C, Roura E, et al. (2007) Absorption and pharmacokinetics of grapefruit flavanones in beagles. Br J Nutr 98, 86-92.

20. Baker TR, Wehmeyer KR, Kelm GR, et al. (1995) Development and application of a gas chromatographic/mass spectrometric/mass spectrometric method for the determination of 5-methoxyflavone in rat and dog plasma. J Mass Spectrom 30, 438-445.

21. Aura AM, O'Leary KA, Williamson G, et al. (2002) Quercetin derivatives are deconjugated and converted to hydroxyphenylacetic acids but not methylated by human fecal flora in vitro. J Agric Food Chem 50, 1725-1730.

22. Cermak R, Breves G, Lüpke M, et al. (2006) In vitro degradation of the flavonol quercetin and of quercetin glycosides in the porcine hindgut. Arch Anim Nutr 60, 180-189.

23. Drochner W \& Meyer H (1991) Digestion of organic matter in the large intestine of ruminants, horses, pigs and dogs. J Anim Physiol Anim Nutr 65, 18-40.

24. Davies B \& Morris T (1993) Physiological parameters in laboratory animals and humans. Pharm Res 10, 1093-1095.

25. Sharer JE, Shipley LA, Vandenbranden MR, et al. (1995) Comparisons of phase I and phase II in vitro hepatic enzyme activities of human, dog, rhesus monkey, and cynomolgus monkey. Drug Metab Dispos 23, 1231-1241.

26. Prueksaritanont T, Gorham LM, Hochman JH, et al. (1996) Comparative studies of drug-metabolizing enzymes in dog, monkey, and human small intestines, and in Caco-2 cells. Drug Metab Dispos 24, 634-642.

27. Mullen W, Rouanet JM, Auger C, et al. (2008) Bioavailability of $\left[2-{ }^{14} \mathrm{C}\right]$ quercetin- $4^{\prime}$-glucoside in rats. J Agric Food Chem 56, $12127-12137$.

28. Gu L, Laly M, Chang HC, et al. (2005) Isoflavone conjugates are underestimated in tissues using enzymatic hydrolysis. J Agric Food Chem 53, 6858-6863.

29. National Toxicology Program (1992) Toxicology and carcinogenesis studies of quercetin (CAS no. 117-39-5) in F344 rats (feed studies). Natl Toxicol Program Tech Rep Ser 409, 1-171. 